\title{
Reachability Results in Plane Trees
}

\author{
Albert Oloo Nyariaro ${ }^{1}$, Isaac Owino Okoth ${ }^{2 *}$
}

\begin{abstract}
In this paper, we obtain closed formulas for the number of reachable vertices in labelled plane trees by paths lengths, sinks, leaf sinks, first children, left most path, non-first children, and non-leaves. Our counting objects are plane trees having their edges oriented from a vertex of lower label towards a vertex of higher label. For each statistic, we obtain the average number of reachable vertices. Moreover, we obtain a counting formula for the number of plane trees on $n$ vertices such that exactly $k \leq n$ are reachable from the root.

Keywords: Plane trees, Reachability, Leaf, Sink, First child

2010 AMS: Primary 05A19, 05C05, 05C30
\end{abstract}

${ }_{1}^{1}$ Department of Pure and Applied Mathematics, Maseno University, Maseno, Kenya, ORCID: 0000-0002-2997-0241

${ }^{2}$ Department of Pure and Applied Mathematics, Maseno University, Maseno, Kenya, ORCID: 0000-0003-4503-4733

*Corresponding author: ookoth@maseno.ac.ke

Received: 12 May 2021, Accepted: 25 June 2021, Available online: 30 June 2021

\section{Introduction}

Plane trees (or ordered trees) have been studied extensively in the literature. These trees on $n$ vertices are counted by the $(n-1)^{\text {th }}$ Catalan number

$$
C_{n-1}=\frac{1}{n}\left(\begin{array}{c}
2 n-2 \\
n-1
\end{array}\right) \text {. }
$$

So if the vertices are labelled with labels $1,2, \ldots, n$ then the total number of these trees is $n ! C_{n-1}$. A vertex $j$ is reachable from a vertex $i$ if there is a sequence of oriented edges (paths) from vertex $i$ to vertex $j$, and a path is of length $\ell$ if there are $\ell$ edges on the path. In this context, degree of a vertex is the number of edges that come out of a vertex if the edges are oriented away from the root. A vertex in which there is no edge that is oriented away from it is called a sink whereas a leaf sink is a vertex with only one edge oriented towards it but no edge oriented away from it. The vertices with the same parent are called siblings. Since the siblings are linearly ordered, they are always drawn in a left-to-right pattern where the leftmost sibling is referred to as first child. At a given level $\ell$, the left most child is the eldest child. A left most path refers to a sequence of edges joining eldest children at each level in a plane tree. In this work, we examine the number of reachable vertices from a given root $i$. We also determine a formula for the number of labelled ordered trees on $n$ vertices such that exactly $k$ vertices are reachable from the root. Plane trees considered here have their edges oriented from a vertex of lower label towards a vertex of higher label. This orientation was introduced in [1]. Equivalent results for $t$-ary trees have been obtained by the present authors in [3]. We will now refer to these plane trees simply as trees. In Section 2, we use path lengths to count the trees. The number of sinks and leaf sinks are the statistics used in Section 3 while in Section 4, we use left most paths and first children. We enumerate trees by non-first children and non-leaves in Section 5. We use mainly generating functions and Lagrange Inversion Formula [5] to prove our results. In most cases, we give asymptotic results as well. Lastly, in Section 6 we give a bijective proof of a formula for the number of trees in which a given number of vertices is reachable from the root. 


\section{Enumeration by path lengths}

In the sequel, we enumerate trees by path lengths. We start by proving the main result of this section.

Theorem 2.1. The number of trees on $n$ vertices rooted at vertex $i$ such that vertex $j$ of degree $d$ is reachable from the root in $\ell$ steps is given by

$$
(n-\ell-1) ! \frac{2 \ell+d}{n+\ell-1}\left(\begin{array}{c}
j-i-1 \\
\ell-1
\end{array}\right)\left(\begin{array}{c}
2 n-d-3 \\
n+\ell-2
\end{array}\right) .
$$

Proof. Let $P(x)$ be the generating function for plane trees where $x$ marks the number of non-root vertices. Consider a plane tree rooted at vertex $i$ such that there is a path of length $\ell$ starting at vertex $i$ and ending at vertex $i+\ell$ of degree $d$. This path decomposes the tree into left and right plane subtrees upto length $\ell$. Thus $(P(x) x P(x))^{\ell}$ is the generating function for the number of the trees with a path of length $\ell$ starting at $i$ and ending at $i+\ell$. Vertex $i+\ell$ is joined to $d$ other vertices which are connected to other plane trees hence we have $x(x P(x))^{d}$, to represent the vertex and the subtrees of its children. Putting everything together, we obtain $(P(x) x P(x))^{\ell} x(x P(x))^{d}=x^{\ell+d+1} P(x)^{2 \ell+d}$ as the generating function of the unlabelled plane tree rooted at vertex $i$ with a path of length $\ell$ starting at $i$ and ending at vertex $i+\ell$ of degree $d$. The decomposition is represented by Figure 2.1.

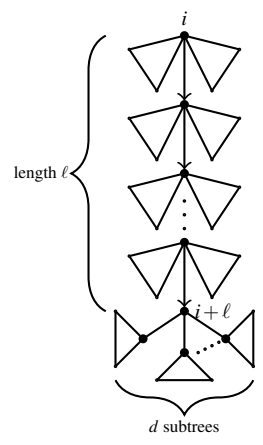

Figure 2.1. Unlabelled plane tree with path length $\ell$.

The generating function for unlabelled plane trees is $P(x)=\frac{1}{1-P(x)}$. We let $x P(x)=F(x)$ so that $F(x)=\frac{x}{1-F(x)}$. Applying Lagrange Inversion Formula, we get

$$
\begin{aligned}
{\left[x^{n}\right] x^{\ell+d+1} P(x)^{2 \ell+d} } & =\left[x^{n}\right] x^{-\ell+1} F(x)^{2 \ell+d} \\
& =\left[x^{n+\ell-1}\right] F(x)^{2 \ell+d} \\
& =\frac{2 \ell+d}{n+\ell-1}\left[t^{n-\ell-d-1}\right](1-t)^{-(n+\ell-1)} \\
& =\frac{2 \ell+d}{n+\ell-1}\left[t^{n-\ell-d-1}\right] \sum_{i \geq 0}\left(\begin{array}{c}
-(n+\ell-1) \\
i
\end{array}\right)(-t)^{i} \\
& =\frac{2 \ell+d}{n+\ell-1}\left[t^{n-\ell-d-1}\right] \sum_{i \geq 0}\left(\begin{array}{c}
n+\ell+i-2 \\
i
\end{array}\right) t^{i} \\
& =\frac{2 \ell+d}{n+\ell-1}\left(\begin{array}{c}
2 n-d-3 \\
n-\ell-d-1
\end{array}\right) .
\end{aligned}
$$

This formula counts the number of unlabelled plane trees in which vertex $i+\ell$ of degree $d$ is reachable from the root in $\ell$ steps. The number of ways of choosing a path of length $\ell$ from vertex $i$ to vertex $j$ is $\left(\begin{array}{c}j-i-1 \\ \ell-1\end{array}\right)$. Once the $\ell+1$ vertices on the path are labelled, there are $(n-\ell-1)$ ! choices for labelling the remaining vertices. Thus, the number of plane trees in which vertex $j$ of degree $d$ is reachable from root $i$ in $\ell$ steps is given by

$$
(n-\ell-1) ! \frac{2 \ell+d}{n+\ell-1}\left(\begin{array}{c}
j-i-1 \\
\ell-1
\end{array}\right)\left(\begin{array}{c}
2 n-d-3 \\
n+\ell-2
\end{array}\right) .
$$

This completes the proof. 
Setting $\ell=0$ in the just proved theorem, we get that there are

$$
(n-1) ! \frac{d}{n-1}\left(\begin{array}{c}
2 n-d-3 \\
n-2
\end{array}\right)=d(n-2) !\left(\begin{array}{c}
2 n-d-3 \\
n-2
\end{array}\right)
$$

trees in which a root of any label has degree $d$.

Quite a number of corollaries of Theorem 2.1 follow:

Corollary 2.2. The total number of trees on $n$ vertices rooted at vertex $i$ such that vertex $j$ is reachable from the root in $\ell$ steps is given by:

$$
(n-\ell-1) ! \frac{2 \ell+1}{2 n-1}\left(\begin{array}{c}
j-i-1 \\
\ell-1
\end{array}\right)\left(\begin{array}{c}
2 n-1 \\
n+\ell
\end{array}\right) .
$$

Proof. The result follows by summing over all $d$ in Theorem 2.1 .

By summing over all $j$ in Equation (2.1), we get that

Corollary 2.3. The number of vertices in trees of order $n$ that are reachable from root $i$ in $\ell$ steps is given by

$$
(n-\ell-1) ! \frac{2 \ell+1}{2 n-1}\left(\begin{array}{c}
n-i \\
\ell
\end{array}\right)\left(\begin{array}{c}
2 n-1 \\
n+\ell
\end{array}\right) .
$$

Moreover, summing over all $i$ in Equation (2.2), we get the total number of reachable vertices:

Corollary 2.4. There are a total of

$$
(n-\ell-1) ! \frac{2 \ell+1}{2 n-1}\left(\begin{array}{c}
n \\
\ell+1
\end{array}\right)\left(\begin{array}{c}
2 n-1 \\
n+\ell
\end{array}\right)
$$

vertices that are reachable from the root in $\ell$ steps, in trees with $n$ vertices.

Now, summing over all $\ell$ in Equation (2.3) we obtain

Corollary 2.5. The total number of vertices in trees on $n$ vertices that are reachable from the root is given by

$$
\frac{n !}{2 n-1} \sum_{\ell=0}^{n-1} \frac{2 \ell+1}{(\ell+1) !}\left(\begin{array}{c}
2 n-1 \\
n+\ell
\end{array}\right) \text {. }
$$

Corollary 2.6. On average, the number of vertices that are reachable from the root in $\ell$ steps in a random tree is $\frac{2 \ell+1}{(\ell+1) !}$.

Proof. Dividing the total number of vertices that are reachable from the root in $\ell$ steps in plane trees (See Equation (2.3)) by the total number of labelled plane trees, we get

$$
A=\frac{(n-\ell-1) ! \frac{2 \ell+1}{2 n-1}\left(\begin{array}{c}
n \\
\ell+1
\end{array}\right)\left(\begin{array}{c}
2 n-1 \\
n-\ell-1
\end{array}\right)}{(n-1) !\left(\begin{array}{c}
2 n-2 \\
n-1
\end{array}\right)}
$$

as the average number of vertices that are reachable in $\ell$ steps from the root in trees with $n$ vertices. We simplify the average to get

$$
A=\frac{2 \ell+1}{(l+1) !} \frac{(n-1) ! n !}{(n+\ell) !(n-\ell-1) !} .
$$

Now, taking limits as $n \rightarrow \infty$, we get

$$
\begin{aligned}
\lim _{n \rightarrow \infty} A & =\frac{2 \ell+1}{(\ell+1) !} \lim _{n \rightarrow \infty} \frac{(n-1) ! n !}{(n+\ell) !(n-\ell-1) !} \\
& =\frac{2 \ell+1}{(\ell+1) !} \lim _{n \rightarrow \infty} \frac{(n-1)(n-2)(n-3) \cdots(n-\ell)}{(n+\ell)(n+\ell-1) \cdots(n+1)} \\
& =\frac{2 \ell+1}{(\ell+1) !} \lim _{n \rightarrow \infty}\left(\frac{n^{\ell}+\cdots}{n^{\ell}+\cdots}\right) \\
& =\frac{2 \ell+1}{(\ell+1) !} .
\end{aligned}
$$

Hence the desired result. 
Corollary 2.7. The number of trees of order $n$ in which there is a path of length $\ell$ starting at the root and ending at a vertex of degree $d$ is given by

$$
(n-\ell-1) ! \frac{2 \ell+d}{n+\ell-1}\left(\begin{array}{c}
n \\
\ell+1
\end{array}\right)\left(\begin{array}{c}
2 n-d-3 \\
n+\ell-2
\end{array}\right) .
$$

Proof. We obtain the result by summing over all $i$ and $j$ in Theorem 2.1.

Setting $\ell=0$ in Equation (2.4), we get

$$
\frac{n ! d}{n-1}\left(\begin{array}{c}
2 n-d-3 \\
n-2
\end{array}\right)
$$

as the formula which counts the number of trees on $n$ vertices such that the root is of degree $d$. Also setting $\ell=1$ in Equation (2.4), we obtain

$$
\frac{(d+2)(n-1) !}{2}\left(\begin{array}{c}
2 n-d-3 \\
n-1
\end{array}\right)
$$

This formula counts the total number of children of degree $d$, in all trees of order $n$.

\section{Enumeration by sinks and leaf sinks}

In this section, we enumerate trees with respect to sinks and leaf sinks.

Proposition 3.1. The number of trees of order $n$ in which vertex $j$, a sink of degree $d$, is reachable from a root $i$ in $\ell$ steps is given by

$$
(n-\ell-d-1) ! \frac{2 \ell+d}{n+\ell-1}\left(\begin{array}{c}
j-i-1 \\
\ell-1
\end{array}\right)\left(\begin{array}{c}
j-\ell-1 \\
d
\end{array}\right)\left(\begin{array}{c}
2 n-d-3 \\
n+\ell-2
\end{array}\right) .
$$

Proof. From the proof of Theorem 2.1, it follows that there are

$$
\frac{2 \ell+d}{n+\ell-1}\left(\begin{array}{c}
2 n-d-3 \\
n+\ell-2
\end{array}\right)
$$

unlabelled trees with a path of length $\ell$ starting at a root $i$ and terminating at vertex $i+\ell$ of degree $d$. Now, consider a path of length $\ell$ starting at root $i$ and ending at vertex $j$. There are $\left(\begin{array}{c}j-i-1 \\ \ell-1\end{array}\right)$ such paths. Since vertex $j$ is a sink of degree $d$, the labels of the $d$ vertices must be less than $j$. Thus there are $\left(\begin{array}{c}j-i-1 \\ d\end{array}\right)$ choices for the labels. Once the $\ell+1$ vertices on the path and the $d$ children of $j$ are labelled, there are $(n-\ell-d-1)$ ! choices for the other labels in the tree. Collecting everything, we arrive at the required formula.

As seen in the previous section, a number of corollaries follow. We obtain the following result by summing over all $j$ in Equation (3.1).

Corollary 3.2. The total number of sinks of degree $d$ that are reachable, in $\ell$ steps, from the root $i$ in trees with $n$ vertices is given by

$$
(n-\ell-d-1) ! \frac{2 \ell+d}{n+\ell-1} \sum_{j=\ell+i}^{n}\left(\begin{array}{c}
j-i-1 \\
\ell-1
\end{array}\right)\left(\begin{array}{c}
j-\ell-1 \\
d
\end{array}\right)\left(\begin{array}{c}
2 n-d-3 \\
n+\ell-2
\end{array}\right) .
$$

Moreover, setting $\ell=0$ and $j=i$ in Equation (3.1) we obtain that the total number of trees with $n$ vertices such that root $i$ is a sink of degree $d$ is given by

$$
(n-d-1) ! \frac{d}{n-1}\left(\begin{array}{c}
i-1 \\
d
\end{array}\right)\left(\begin{array}{c}
2 n-d-3 \\
n-2
\end{array}\right)
$$

and also by setting setting $\ell=1$ in Equation (3.1), we get that there are

$$
(n-d-2) ! \frac{2+d}{n}\left(\begin{array}{c}
j-2 \\
d
\end{array}\right)\left(\begin{array}{c}
2 n-d-3 \\
n-1
\end{array}\right)
$$

children of the root labelled $j$ having degree $d$ in trees on $n$ vertices.

Summing over all $j$ in Equation (3.3) we obtain the number of children of the root, which are also sinks of degree $d$, in trees of order $n$. By summing over all $i$ in Equation (3.2), we obtain 
Corollary 3.3. The total number of trees of order $n$ with root sinks of degree $d$ is given by:

$$
(n-d-1) ! \frac{d}{n-1}\left(\begin{array}{c}
n \\
d+1
\end{array}\right)\left(\begin{array}{c}
2 n-d-3 \\
n-2
\end{array}\right) .
$$

Next, we find an asymptotic result:

Corollary 3.4. On average there are

$$
\frac{d}{2^{d+1}(d+1) !}
$$

root sinks of degree $d$ in a random tree.

Proof. Diving the total number of labelled plane trees of order $n$ with root sinks of degree $d$ (Equation (3.4)), by the total number of labelled plane trees we get,

$$
\frac{(n-d-1) ! \frac{d}{n-1}\left(\begin{array}{c}
n \\
d+1
\end{array}\right)\left(\begin{array}{c}
2 n-d-3 \\
n-2
\end{array}\right)}{(n-1) !\left(\begin{array}{c}
2 n-2 \\
n-1
\end{array}\right)},
$$

as the average number of root sinks of degree $d$ in a random plane tree on $n$ vertices. Simplifying the average and tending $n$ to infinity, we obtain the required result.

Setting $d=0$ in Equation (3.5) we get that the average number of root sinks of degree 0 is zero. This implies that there is no leaf sink which is also a root. For the remainder of this section, we enumerate the trees by leaf sinks.

Proposition 3.5. The total number of trees of order $n$ in which vertex $j$, a leaf sink, is reachable from a root $i$ in $\ell$ steps is given by the formula,

$$
(n-\ell-1) ! \frac{\ell}{n-1}\left(\begin{array}{c}
j-i-1 \\
\ell-1
\end{array}\right)\left(\begin{array}{c}
2 n-2 \\
n+\ell-1
\end{array}\right) .
$$

Proof. The result follows by setting $d=0$ in Proposition 3.1. However, to show the decomposition we will construct the proof. Let $P(x)$ to be the generating function for plane trees where $x$ is marking a non-root vertex. Consider a plane tree rooted at vertex $i$ such that there is a path of length $\ell$ starting at vertex $i$ and terminating at a vertex of label $i+\ell$ which is also a leaf sink. The path decomposes the tree into left and right plane subtrees upto length $\ell$. See Figure 3.1.

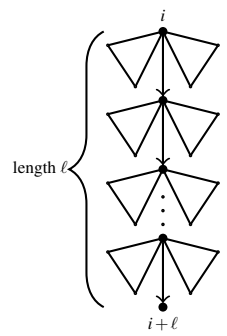

Figure 3.1. Unlabelled plane tree with path length $\ell$ with vertex $i+\ell$ as a leaf sink.

Vertex $i+\ell$ is not connected to any other tree thus vertex is represented by $x$ in the generating function. So we have $x(P(x) x P(x))^{\ell}=x\left(x P(x)^{2}\right)^{\ell}$ as the generating function of the unlabelled trees rooted at vertex $i$ with a path of length $\ell$ starting at the root and ending at a leaf sink $i+\ell$. The generating function for the number of unlabelled plane trees satisfies $P(x)=\frac{1}{1-x P(x)}$. We set $x P(x)=F(x)$ so that $F(x)=\frac{x}{1-F(x)}$. By Lagrange Inversion Formula, we obtain

$$
\begin{aligned}
{\left[x^{n}\right] x\left(x P(x)^{2}\right)^{\ell}=\left[x^{n+\ell-1}\right] F(x)^{2 \ell} } & =\frac{2 \ell}{n+\ell-1}\left[t^{n-\ell-1}\right]\left((1-t)^{-(n+\ell-1)}\right) \\
& =\frac{2 \ell}{n+\ell-1}\left[t^{n-\ell-1}\right] \sum_{i \geq 0}\left(\begin{array}{c}
-(n+\ell-1) \\
i
\end{array}\right)(-t)^{i}
\end{aligned}
$$


which reduces to

$$
\left[x^{n}\right] x\left(x P(x)^{2}\right)^{\ell}=\frac{\ell}{n-1}\left(\begin{array}{c}
2 n-2 \\
n+\ell-1
\end{array}\right) .
$$

This formula counts the number of unlabelled plane trees with a path of length $\ell$ starting at a root $i$ and ending at a leaf sink $i+\ell$. Consider a path of length $\ell$ starting at vertex $i$ and ending at vertex $j$. There are $\left(\begin{array}{c}j-i-1 \\ \ell-1\end{array}\right)$ possible paths. Once the $\ell+1$ vertices on the path have been labelled, there are $(n-\ell-1)$ ! ways of labelling the remaining vertices. Therefore, the total number of labelled plane trees of order $n$ in which vertex $j$ is a leaf sink reachable from vertex $i$ in $\ell$ steps is

$$
(n-\ell-1) ! \frac{\ell}{n-1}\left(\begin{array}{c}
j-i-1 \\
\ell-1
\end{array}\right)\left(\begin{array}{c}
2 n-2 \\
n+\ell-1
\end{array}\right) \text {. }
$$

This completes the proof.

We obtain the following result by summing over all $j$ in Equation (3.6).

Corollary 3.6. The total number of leaf sinks that are reachable, in $\ell$ steps, from root $i$ in trees with $n$ vertices is given by

$$
(n-\ell-1) ! \frac{\ell}{n-1}\left(\begin{array}{c}
n-i \\
\ell
\end{array}\right)\left(\begin{array}{c}
2 n-2 \\
n+\ell-1
\end{array}\right) .
$$

Corollary 3.7. There are

$$
(n-\ell-1) ! \frac{\ell}{n-1}\left(\begin{array}{c}
n \\
\ell+1
\end{array}\right)\left(\begin{array}{c}
2 n-2 \\
n+\ell-1
\end{array}\right),
$$

leaf sinks at step $\ell$ that are reachable from the root in trees with $n$ vertices.

Proof. The result is evident by summing over all $i$ in Equation (3.7).

The formula below follows by summing over all $\ell$ in Equation (3.8).

Corollary 3.8. The formula for the number of leaf sinks in trees of order $n$ that are reachable from the root is

$$
\frac{n !}{n-1} \sum_{\ell=0}^{n-1} \frac{\ell}{(\ell+1) !}\left(\begin{array}{c}
2 n-2 \\
n+\ell-1
\end{array}\right) .
$$

Corollary 3.9. The average number of leaf sinks that are reachable from the root in $\ell$ steps in a random tree is

$$
\frac{\ell}{(\ell+1) !} \text {. }
$$

Proof. The result follows by dividing the total number of leaf sinks that are reachable from the root in a labelled plane tree, i.e Equation (3.8), by the total number of labelled plane trees, and tending $n \rightarrow \infty$.

\section{Enumeration by left most paths and first children}

In this section, we continue our investigation of reachable vertices but now according to lengths of left most paths and first children. We begin by left most paths. Recall that a left most path refers to a path that joins the eldest children at each level in a plane tree.

Proposition 4.1. The number of trees of order $n$ in which there is a left most path of length $\ell$ from a root $i$ to a vertex $j$ is given by

$$
(n-\ell-1) ! \frac{\ell+1}{n}\left(\begin{array}{c}
j-i-1 \\
\ell-1
\end{array}\right)\left(\begin{array}{c}
2 n-\ell-2 \\
n-\ell-1
\end{array}\right) .
$$


Proof. Let $P(x)$ be the generating function for plane trees. Here again, $x$ marks vertices in unrooted plane trees. Figure 4.1 gives the decomposition of these trees by left most path.

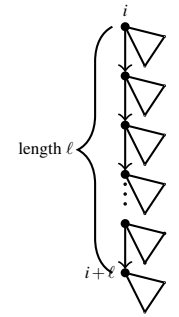

Figure 4.1. Unlabelled plane tree with left most path of length $\ell$.

The decomposition shows that $(x P(x))^{\ell+1}=x^{\ell+1} P(x)^{\ell+1}$ is the generating function for the number of unlabelled trees in which there is a left most path of length $\ell$. It now remains to extract the coefficient of $x^{n}$ in the generating function $x^{\ell+1} P(x)^{\ell+1}$. We set $x P(x)=F(x)$ so that $F(x)=\frac{x}{1-F(x)}$ and by Lagrange Inversion Formula we get

$$
\begin{aligned}
{\left[x^{n}\right](x P(x))^{\ell+1} } & =\left[x^{n}\right] F(x)^{\ell+1} \\
& =\frac{\ell+1}{n}\left[t^{n-\ell-1}\right](1-t)^{-n} \\
& =\frac{\ell+1}{n}\left[t^{n-\ell-1}\right] \sum_{k \geq 0}\left(\begin{array}{c}
-n \\
k
\end{array}\right)(-t)^{k} \\
& =\frac{\ell+1}{n}\left[t^{n-\ell-1}\right] \sum_{k \geq 0}\left(\begin{array}{c}
n-1+k \\
k
\end{array}\right) t^{k} \\
& =\frac{\ell+1}{n}\left(\begin{array}{c}
2 n-\ell-2 \\
n-\ell-1
\end{array}\right) .
\end{aligned}
$$

There are $\left(\begin{array}{c}j-i-1 \\ \ell-1\end{array}\right)$ choices for paths of length $\ell$ between vertices $i$ and $j$. After the $\ell+1$ vertices on the path have been labelled, by choice of paths, the remaining vertices are labelled in $(n-\ell-1)$ ! ways. Therefore, we find that the number of trees of order $n$ in which there is a left most path of length $\ell$ is given by

$$
(n-\ell-1) ! \frac{\ell+1}{n}\left(\begin{array}{c}
j-i-1 \\
\ell-1
\end{array}\right)\left(\begin{array}{c}
2 n-\ell-2 \\
n-\ell-1
\end{array}\right) .
$$

Thus the proof.

By summing over all $j$ in Equation (4.1) we find that there are

$$
(n-\ell-1) ! \frac{\ell+1}{n}\left(\begin{array}{c}
n-i \\
\ell
\end{array}\right)\left(\begin{array}{c}
2 n-\ell-2 \\
n-\ell-1
\end{array}\right)
$$

trees on $n$ vertices in which there is a left most path of length $\ell$ from root $i$. Also, summing over all $i$ in Equation (4.2), we obtain the formula for the number of trees of order $n$ in which there is a left most path of length $\ell$ from the root as

$$
(n-\ell-1) ! \frac{\ell+1}{n}\left(\begin{array}{c}
n \\
\ell+1
\end{array}\right)\left(\begin{array}{c}
2 n-\ell-2 \\
n-\ell-1
\end{array}\right) .
$$

Setting $\ell=0$ in Equation (4.3) we rediscover the formula for the number of labelled plane trees, that is $n ! C_{n-1}$ where $C_{n}$ is the $n^{\text {th }}$ Catalan number.

Corollary 4.2. The average number of eldest children at length $\ell$ from the root in a random tree is $\frac{1}{\ell ! 2^{\ell}}$.

Proof. As before, we divide the total number of labelled plane trees of order $n$ in which there is a left most path of length $\ell$, Equation (4.3), by the total number of labelled plane trees. We then simplify the resultant and tend $n \rightarrow \infty$.

We now switch our attention to leaf sinks and left most paths. 
Proposition 4.3. The number of trees of order $n$ rooted at vertex $i$ in which there is a left most path of length $\ell$ such that the final vertex $j$ is a leaf sink is given by

$$
(n-\ell-1) ! \frac{\ell}{n-1}\left(\begin{array}{c}
j-i-1 \\
\ell-1
\end{array}\right)\left(\begin{array}{c}
2 n-\ell-3 \\
n-\ell-1
\end{array}\right) .
$$

Proof. Let $P(x)$ to be the generating function for plane trees where $x$ is marking a non-root vertex. Consider a plane tree rooted at vertex $i$ such that there is a left most path of length $\ell$ starting at vertex $i$ and ending at vertex $i+\ell$ which is also a leaf sink. The path decomposes the tree into right plane subtrees upto length $\ell$. See Figure 4.2.

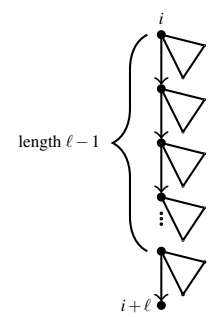

Figure 4.2. Unlabelled plane tree with left most path of length $\ell$ and the final vertex is a leaf sink.

Vertex $i+\ell$ is not connected to any other subtree. So we have $x(x P(x))^{\ell}=x^{\ell+1} P(x)^{\ell}$ as the generating function of the unlabelled plane tree rooted at vertex $i$ with a left most path of length $\ell$ starting at the root and ending at leaf sink $i+\ell$. We set $x P(x)=F(x)$ and apply Lagrange Inversion Formula, to get

$$
\begin{aligned}
{\left[x^{n}\right] x^{\ell+1} P(x)^{\ell} } & =\left[x^{n}\right] x F(x)^{\ell}=\left[x^{n-1}\right] F(x)^{\ell} \\
& =\frac{\ell}{n-\ell}\left[t^{n-\ell-1}\right](1-t)^{-(n-1)} \\
& =\frac{\ell}{n-\ell}\left[t^{n-\ell-1}\right] \sum_{k \geq 0}\left(\begin{array}{c}
-(n-1) \\
k
\end{array}\right)(-t)^{k} \\
& =\frac{\ell}{n-1}\left(\begin{array}{c}
2 n-\ell-3 \\
n-\ell-1
\end{array}\right),
\end{aligned}
$$

as the formula for the number of unlabelled plane trees with a left most path of length $\ell$ starting at a root and ending at a leaf sink. Consider a path of length $\ell$ starting at vertex $i$ and ending at vertex $j$. There are $\left(\begin{array}{c}j-i-1 \\ \ell-1\end{array}\right)$ possible paths. Once the $\ell+1$ vertices on the path have been labelled, there are $(n-\ell-1)$ ! ways of labelling the remaining vertices. Therefore, putting everything together we obtain the desired formula.

By summing over all $j$ in Equation (4.4) we get the total number of trees on $n$ vertices in which there is a left most path of length $\ell$ from root $i$ and a final vertex is a leaf sink as

$$
(n-\ell-1) ! \frac{\ell}{n-1}\left(\begin{array}{c}
n-i \\
\ell
\end{array}\right)\left(\begin{array}{c}
2 n-\ell-3 \\
n-\ell-1
\end{array}\right) .
$$

Also, by summing over all $i$ in Equation (4.5) we get that the total number of trees on $n$ vertices in which there is a left most path of length $\ell$ from the root and the final vertex is a leaf sink as

$$
(n-\ell-1) ! \frac{\ell}{n-1}\left(\begin{array}{c}
n \\
\ell+1
\end{array}\right)\left(\begin{array}{c}
2 n-\ell-3 \\
n-\ell-1
\end{array}\right) .
$$

Moreover, if we sum over all $\ell$ in Equation (4.6) and then simplify, we obtain the formula for number of trees of order $n$ in which there is a left most path starting from the root and the ending vertex is a leaf sink:

$$
(n-2) ! \sum_{\ell=0}^{n-1} \frac{\ell}{(\ell+1) !}\left(\begin{array}{c}
2 n-\ell-3 \\
n-\ell-1
\end{array}\right)
$$

Corollary 4.4. On average, there are $\frac{1}{(\ell+1) ! 2^{\ell+1}}$ eldest children which are also leaf sinks in random tree. 
Proof. We divide the total number of labelled plane trees in which there is a left most path of length $\ell$ from the root and the final vertex is a leaf sink (See Equation (4.6)), by the total number of labelled plane trees to obtain

$$
\frac{(n-\ell-1) ! \frac{\ell}{n-1}\left(\begin{array}{c}
n \\
\ell+1
\end{array}\right)\left(\begin{array}{c}
2 n-\ell-3 \\
n-\ell-1
\end{array}\right)}{(n-1) !\left(\begin{array}{c}
2 n-2 \\
n-1
\end{array}\right)}
$$

as the average number of labelled plane trees in which there is a left most path and a final vertex is a leaf sink. We then simplify and tend $n \rightarrow \infty$ to obtain the desired result.

Recall that in ordered trees, the children (or siblings) are linearly ordered and are drawn in a left-to-right pattern where the left most child is called the first child to the parent. Enumerating the trees by first children we find that,

Proposition 4.5. The number of trees of order $n$ with vertex $i$ as a root and vertex $j$ as a first child reachable from root in $\ell$ steps is given by

$$
(n-\ell-1) ! \frac{\ell}{n-1}\left(\begin{array}{c}
j-i-1 \\
\ell-1
\end{array}\right)\left(\begin{array}{c}
2 n-2 \\
n+\ell-1
\end{array}\right) .
$$

Proof. Let $P(x)$ be the generating function for the plane trees where $x$ represents non-root vertices. Consider a plane tree rooted at vertex $i$ such that there is a path of length $\ell$ starting at vertex $i$ and terminating at vertex $i+\ell$ which is a first child. The path decomposes the tree into left and right plane subtrees upto vertex $\ell-1$. See Figure 4.3.

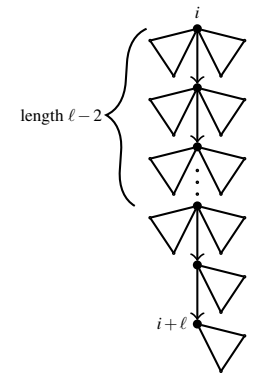

Figure 4.3. Unlabelled plane tree of order $n$ with first child at length $\ell$.

Since vertex $i+\ell$, which is the $(\ell+1)^{\text {th }}$ vertex, is a first child it's parent has no left subtree. Vertex $i+\ell$ can either have children or not. Thus the decomposition gives $\left(x\left(P(x)^{2}\right)^{\ell-1} x P(x) x P(x)=x^{\ell+1} P(x)^{2 \ell}\right.$ as the generating function for unlabelled plane trees rooted at vertex $i$ such that there is a path of length $\ell$ starting at the root and ending at a first child $i+\ell$.

Since $P(x)=1 /(1-x P(x))$, we set $x P(x)=F(x)$ and apply Lagrange Inversion Formula, to obtain

$$
\begin{aligned}
{\left[x^{n}\right]\left(x^{\ell+1} P(x)^{2 \ell}\right) } & =\left[x^{n+\ell-1}\right] F(x)^{2 \ell} \\
& =\frac{2 \ell}{n+\ell-1}\left[t^{n-\ell-1}\right](1-t)^{-(n+\ell-1)} \\
& =\frac{2 \ell}{n+\ell-1}\left[t^{n-\ell-1}\right] \sum_{i \geq 0}\left(\begin{array}{c}
n+\ell+i-2 \\
i
\end{array}\right) t^{i} \\
& =\frac{2 \ell}{n+\ell-1}\left(\begin{array}{c}
2 n-3 \\
n+\ell-2
\end{array}\right) \\
& =\frac{\ell}{n-1}\left(\begin{array}{c}
2 n-2 \\
n+\ell-1
\end{array}\right)
\end{aligned}
$$

as the formula for unlabelled plane trees with a path of length $\ell$ starting at a root and terminating at a first child. The formula then follows by choosing the choices of paths between $i$ and $j$, and labelling the vertices which are not on the path.

By summing over all $j$ in Equation (4.6), we see that the number of first children that are reachable from root $i$, at length $\ell$, in trees on $n$ vertices is given by

$$
(n-\ell-1) ! \frac{\ell}{n-1}\left(\begin{array}{c}
n-i \\
\ell
\end{array}\right)\left(\begin{array}{c}
2 n-2 \\
n+\ell-1
\end{array}\right) .
$$


In addition, we have: The number of first children at level $\ell$ in trees of order $n$ that are reachable from the root is given by

$$
(n-\ell-1) ! \frac{\ell}{n-1}\left(\begin{array}{c}
n \\
\ell+1
\end{array}\right)\left(\begin{array}{c}
2 n-2 \\
n+\ell-1
\end{array}\right) .
$$

This is arrived at by summing over all $i$ in Equation (4.7). Also, by summing over all $\ell$ in Equation (4.8) and simplifying, we obtain the formula for the total number of first children that are reachable from the root in trees of order $n$ :

$$
\frac{n !}{n-1} \sum_{\ell=0}^{n-1} \frac{\ell}{n-1}\left(\begin{array}{c}
2 n-2 \\
n+\ell-1
\end{array}\right)
$$

Corollary 4.6. The average number of first children that are reachable from the root in $\ell$ steps in a random tree is $\frac{\ell}{(\ell+1) !}$.

Proof. By dividing the total number of first children in a labelled plane tree that are reachable from the root (See Equation (4.8)), by the total number of labelled plane trees we obtain,

$$
\frac{(n-\ell-1) ! \frac{\ell}{n-1}\left(\begin{array}{c}
n \\
\ell+1
\end{array}\right)\left(\begin{array}{c}
2 n-2 \\
n+\ell-1
\end{array}\right)}{(n-1) !\left(\begin{array}{c}
2 n-2 \\
n-1
\end{array}\right)}
$$

as the average number of first children in a labelled plane tree of order $n$ that are reachable from the root in $\ell$ steps. We tend $n \rightarrow \infty$ to obtain the required result.

Remark 4.7. Unlabelled plane trees with a path of length $\ell$ starting at a root $i$ and terminating at a leaf sink $j$ has similar generating function as unlabelled trees with a path of length $\ell$ starting at a root $i$ and terminating at a first child $j$. Therefore, they pose similar results if we sum over $i, j$ and $\ell$. Asymptotic results are also the same.

Remark 4.8. If the terminal vertex $j$ is a first child which is also a leaf, then the generating function for trees with root $i$ such that there is a path of length $\ell$ from $i$ to $j$ is given as $\left(x P(x)^{2}\right)^{\ell-1} x P(x) x$. Thus there are

$$
\frac{2 \ell-1}{n+\ell-1}\left(\begin{array}{c}
2 n-3 \\
n+\ell-2
\end{array}\right)
$$

such trees on $n$ vertices.

\section{Enumeration by non-first children and non-leaves}

In plane trees, any vertex (child) which is not leftmost child of the parent vertex is called a non-first child. A vertex which is not a leaf is a non leaf. In this section, we enumerate trees with respect to number of non-first children as well as number of non leaves.

Proposition 5.1. The number of trees on $n$ vertices rooted at vertex $i$ and having vertex $j$ as a non-first child which is reachable from $i$, at length $\ell$, is given by

$$
(n-\ell-1) ! \frac{\ell+1}{n-1}\left(\begin{array}{c}
j-i-1 \\
\ell-1
\end{array}\right)\left(\begin{array}{c}
2 n-2 \\
n+\ell
\end{array}\right) .
$$

Proof. We obtain the generating function by considering a plane tree rooted at vertex $i$ with a path of length $\ell$ starting at $i$ and terminating at a non-first child $i+\ell$. The path decomposes the tree into left and right plane subtrees as shown in Figure 5.1.

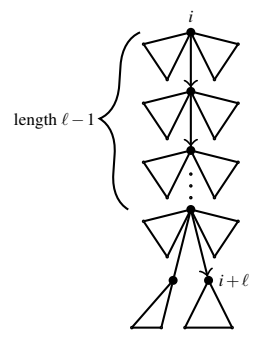

Figure 5.1. Unlabelled plane tree with non-first children at length $\ell$. 
The decomposition gives $x^{\ell+2} P(x)^{2 \ell+2}$ as the generating function for unlabelled plane trees, with a path of length $\ell$ starting at a root and terminating at a non-first child. The generating function for unlabelled plane trees $P(x)=\frac{1}{1-x P(x)}$. We set $x P(x)=F(x)$ and use Lagrange Inversion formula to obtain

$$
\begin{aligned}
{\left[x^{n}\right] x^{\ell+2} P(x)^{2 \ell+2} } & =\left[x^{n+\ell}\right] F(x)^{2 \ell+2}=\frac{2 \ell+2}{n+\ell}\left[t^{n-\ell-2}\right]\left((1-t)^{-1(n+\ell)}\right) \\
& =\frac{2 \ell+2}{n+\ell}\left[t^{n-\ell-2}\right] \sum_{i \geq 0}\left(\begin{array}{c}
n+\ell+i-1 \\
i
\end{array}\right) t^{i} \\
& =\frac{\ell+1}{n-1}\left(\begin{array}{c}
2 n-2 \\
n+\ell
\end{array}\right),
\end{aligned}
$$

as the formula for the number of non-first children that are reachable at length $\ell$ from the root in trees of order $n$. Upon considering the choices for labels on and not on the path, we obtain the desired formula.

By summing over all $j$ in Equation (5.1), we get that the total number of non-first children at level $\ell$ in trees of order $n$ that are reachable from vertex $i$ is

$$
(n-\ell-1) ! \frac{\ell+1}{n-1}\left(\begin{array}{c}
n-i \\
\ell
\end{array}\right)\left(\begin{array}{c}
2 n-2 \\
n+\ell
\end{array}\right) \text {. }
$$

Also, by summing over all $i$ in Equation (5.2), we obtain the following result: The total number of non-first children at level $\ell$ that are reachable from the root in a tree on $n$ vertices is given by

$$
(n-\ell-1) ! \frac{\ell+1}{n-1}\left(\begin{array}{c}
n \\
\ell+1
\end{array}\right)\left(\begin{array}{c}
2 n-2 \\
n+\ell
\end{array}\right) .
$$

Moreover, the total number of non-first children in trees of order $n$ that are reachable from the root is given by

$$
\frac{n !}{n-1} \sum_{\ell=0}^{n-1} \frac{1}{\ell !}\left(\begin{array}{c}
2 n-2 \\
n+\ell
\end{array}\right)
$$

This formula is arrived at by summing over all $\ell$ in Equation (5.3), and simplifying. In a similar fashion as before we have:

Corollary 5.2. The average number of non-first children that are reachable at length $\ell$ from the root in a random tree is given by $\frac{1}{\ell !}$.

For the remainder of this section, we enumerate non-leaf sinks.

Proposition 5.3. The number of trees of order $n$ with vertex $i$ as a root and vertex $j$ as non-leaf, which is reachable from the root at length $\ell$ is given by

$$
(n-\ell-1) ! \frac{\ell+1}{n-1}\left(\begin{array}{c}
j-i-1 \\
\ell-1
\end{array}\right)\left(\begin{array}{c}
2 n-2 \\
n+\ell
\end{array}\right) .
$$

Proof. Consider a tree rooted at vertex $i$ with a path of length $\ell$ from the root to vertex $i+\ell$ which is non-leaf. This path decomposes the tree into left and right subtrees upto step $\ell$. Moreover, since vertex $i+\ell$ is a non-leaf, there must be a subtree of $i+\ell$ which may be empty and a subtree, rooted at a child of $i+\ell$. This subtree may also be empty. The decomposition is therefore given by Figure 5.2.

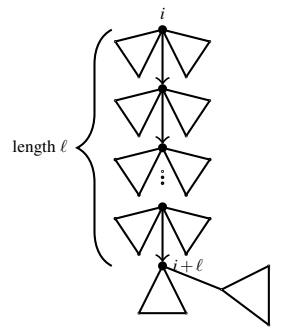

Figure 5.2. Unlabelled plane tree with a non-leaf vertex at length $\ell$. 
The generating function for the trees is thus $\left(x P(x)^{2}\right)^{\ell} x P(x) x P(x)=x^{\ell+2} P(x)^{2 \ell+2}$, where $P(x)$ is the generating function for unlabelled plane trees with $x$ marking non-root vertices. The required result therefore follows by applying Lagrange Inversion formula, upon setting $x P(x)=F(x)$, and giving the number of choices for the labels on the path and those that are not on the path.

Remark 5.4. The number of trees with a non-leaf vertex $j$ which is reachable at length $\ell$ from a root $i$ have similar generating functions (though different decompositions) as for the case of non-first children. The results in the case of non-first children therefore hold for non-leaf vertices.

\section{Enumeration by exact number of vertices}

The number of exact vertices that are reachable from a given vertex has been studied for the case of labelled ordinary trees. Quite a number of results were obtained by Okoth in his PhD thesis, [2]. Similarly, Seo and Shin [4] established a formula for rooted Cayley trees of order $n$ in which there is a maximal increasing subtree of order $k$. In this section, we obtain a formula for the number of ordered trees in which a given number of vertices are reachable from the root.

Theorem 6.1. The total number of trees of order $n$ such that exactly $k$ vertices are reachable from the root is given by

$$
O_{n, k}=\sum_{k \leq m+1 \leq n}\left(\begin{array}{c}
n \\
m+1
\end{array}\right) z_{m, k-1} \frac{m-k+1}{n-k}(n-k)^{(n-m-1)}
$$

for $0 \leq k<n, O_{n, n}=(2 n-3) !$ !, where $n^{(r)}=n(n+1)(n+2) \cdots(n+r-1)$ is a rising factorial and $z_{m, k}$ is the number of ordered trees on $m+1$ vertices with additional $(m-k)$ decreasing leaves attached to an increasing tree with $k$ edges.

A subtree of a rooted tree is said to be increasing if the labels in the subtree are increasing as one moves away from the root. A maximal increasing subtree of a $v$-rooted tree is an increasing subtree rooted at $v$ and having the highest number of vertices. Seo and Shin [4], showed that Equation (6.1) gives the number of ordered trees on $[n]$ with its maximal decreasing subtree having $k$ vertices. Now, orienting the edges of the ordered tree with $n$ vertices from vertices of lower label towards vertices of higher label, we obtain an ordered tree in which exactly $k$ are reachable from the root if its maximal increasing subtree has $k$ vertices. This proves Theorem 6.1 .

Corollary 6.2. The number of trees of order $n$ having exactly $k \geq 2$ vertices reachable from root 1 is given by

$$
(2 k-3) O_{n-1, k-1}
$$

where $O_{n, k}$ is given by Equation (6.1).

Proof. Consider an ordered tree $P$ of order $n-1$ such that the vertices are labelled $2,3, \cdots, n$. Let the root be of label $v_{1}$. Moreover, let the number of vertices that are reachable from $v_{1}$ be exactly $k-1$. We follow the following steps in obtaining trees in which exactly $k$ vertices are reachable from root of label 1 :

Step 1: Let $P_{0}$ be the maximal increasing subtree having vertex set $\left\{v_{1}, v_{2}, \ldots, v_{k-1}\right\}$ where $v_{i}<v_{i+1}$ for all $i$. In $P$, delete all the edges in $P_{0}$ to obtain non-single vertex subtrees $P_{1}, P_{2}, \cdots, P_{m}$.
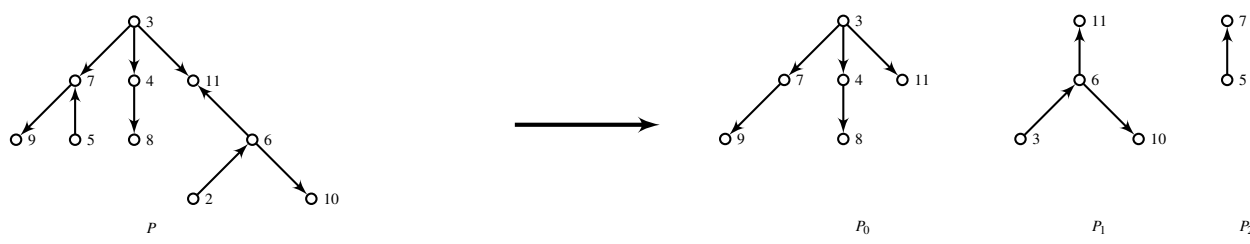

$P$

$P_{0}$

$P_{2}$

Figure 6.1. Diagram showing Step 1 in the proof of Corollary 6.2

Step 2: Relabel the vertices of the maximal increasing subtree $P_{0}$ with the vertex $v_{1}$ now as $1, v_{2}$ as $v_{1}, v_{3}$ as $v_{2}$ and so on. The maximal increasing tree $P_{0}$ still has $k-1$ vertices. There are $2 k-3$ positions in the new maximal increasing subtree rooted at 1 to attach vertex $v_{k-1}$. For each maximal increasing subtree previously rooted at $v_{1}$ we obtain $2 k-3$ new subtrees rooted at vertex 1 with $k$ reachable vertices. 


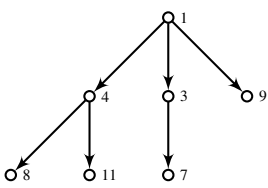

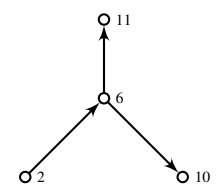

$P_{1}$

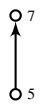

Figure 6.2. Diagram showing Step 2 in the proof of Corollary 6.2

Step 3: Identify vertex $v_{i}$ in the subtrees $P_{1}, P_{2}, \ldots, P_{k-1}$ with vertex $v_{i}$ in the new maximal increasing subtree, for all $i \in\{1, \ldots, k-1\}$. for which $v_{i}$ occurs in one of the $P_{j}$.

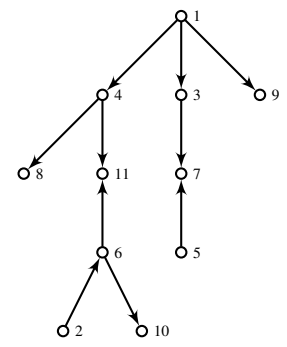

Figure 6.3. Diagram showing Step 3 in the proof of Corollary 6.2

Theorem 6.1, gives the total number of ordered labelled trees of order $n$ such that exactly $k$ vertices are reachable from the root. Now, for maximal increasing subtrees with $k-1$ vertices we substitute $k$ and $n$ in the Equation (6.1) above with $k-1$ and $n-1$ respectively to obtain the formula for the number of labelled trees of order $n$ having exactly $k$ vertices reachable from vertex 1 as

$$
(2 k-3) O_{n-1, k-1} .
$$

Thus the proof is complete.

Corollary 6.3. There are

$$
(2 n-2 i+1) ! !(n-i+1)(n+1)(n+2) \cdots(n+i-2)
$$

trees on $n$ vertices such that exactly $n-i+1$ reachable vertices from root $i$.

Proof. There are $(2 n-2 i+1)$ !! recursive trees on $n-i+1$ vertices (See Lemma 2 in [4]). Since there are $n-(n-i+1)=i-1$ vertices which are not reachable from vertex $i$, then all the $i-1$ vertices have labels less than $i$. The number of ways of adding the $i-1$ vertices to recursive tree successively is given by $(n-i+1)(n+1)(n+2) \cdots(n+i-2)$ (See Lemma 2 in [4]). Therefore the total number of trees on $n$ vertices with exactly $n-i-1$ vertices reachable from vertex $i$ is given by

$$
(2 n-2 i+1) ! !(n-i+1)(n+1)(n+2) \cdots(n+i-2) .
$$

Hence the desired formula.

\section{Acknowledgements}

The authors would like to express their sincere thanks to the editor and the anonymous reviewers for their helpful comments and suggestions.

\section{Funding}

There is no funding for this work. 


\section{Availability of data and materials}

Not applicable.

\section{Competing interests}

The authors declare that they have no competing interests.

\section{Authors' contributions}

All authors contributed equally to the writing of this paper. All authors read and approved the final manuscript.

\section{References}

[1] R. R. X. Du, J. Yin. Counting labelled trees with a given indegree sequence. J. Combin. Theory, Ser. A, 117(3)(2010), 345 353.

[2] I. O. Okoth. Combinatorics of oriented trees and tree-like structures. PhD Thesis, Stellenbosch University, (2015).

[3] I. O. Okoth, A. O. Nyariaro. Reachability results in labelled $t$-ary trees. Preprint, (2021).

[4] S. Seo, H. Shin. On the enumeration of rooted trees with fixed size of maximal decreasing trees. Discrete Math., 312(2) (2012), 419-426.

[5] H. S. Wilf. Generatingfunctionology. A. K. Peters, Ltd., Natick, MA, USA, (2006). 